\title{
Alt werden in Arbeit - wie kann das gelingen?
}

\author{
EVELYN RÄDER
}

Alt werden in Erwerbsarbeit - das könnte durchaus gut gelingen, wenn die Arbeit alters- und alternsgerecht gestaltet würde und Ältere einen besseren Zugang zum Arbeitsmarkt hätten. Aktuell sieht die Lage jedoch anders aus: Viel zu wenige Unternehmen beschäftigen sich mit den Folgen des demografischen Wandels. Und viel zu viele Unternehmen vernachlässigen eine aktive alternsbezogene Personalpolitik. Die relativ geringe Erwerbsbeteiligung von älteren Menschen deutet auf eine nicht alters- und alternsgerechte Arbeitswelt hin. Die Politik ist eher vermittelnd denn gestaltend tätig. Gewerkschaften und betriebliche Interessenvertretungen werden damit zum Ausfallbürgen in diesem Handlungsfeld. Der folgende Beitrag greift die Situation von Älteren am Arbeitsmarkt auf, beschreibt den Handlungsbedarf hinsichtlich der Gestaltung alters- und alternsgerechter Arbeitsbedingungen und gibt einen Einblick in die tarifliche und betriebliche Arbeit zur Gestaltung des demografischen Wandels im Dienstleistungsbereich.

\section{Erwerbsbeteiligung und Arbeitslosigkeit Älterer}

2010 stellten die 50- bis 64-Jährigen rund $30 \%$ der Bevölkerung im erwerbsfähigen Alter in Deutschland. ${ }^{1}$ Dieser Anteil wird sich zwischen 2017 und 2024 auf fast $40 \%$ erhöhen. ${ }^{2}$ Die Erwerbs-, Erwerbstätigen- und Beschäftigungsquoten der 50- bis unter 65-Jährigen haben in Deutschland in den letzten Jahren kontinuierlich zugenommen. Gleichwohl waren in der Gruppe der 60- bis 65-Jährigen 2010 nur $27,5 \%$ und damit nur ein gutes Viertel sozialversicherungspflichtig beschäftigt. ${ }^{3}$ Jugendzentrierung und als Kehrseite Altersdiskriminierung sind nach wie vor sehr verbreitet, wie unter anderem eine jüngst vom Bundesgerichtshof (BGH) entschiedene Klage vor Augen führt: Zur Verhandlung stand der Fall eines 62-jährigen Geschäftsführers einer Krankenhausgesellschaft, dessen Vertragsverhältnis unter Verweis auf sein Alter nicht verlängert werden sollte. ${ }^{4}$

Die Älteren haben zwar die kleinsten Zugangsrisiken in Arbeitslosigkeit, aber gleichzeitig sinken mit zunehmendem Alter die Chancen, Arbeitslosigkeit durch Beschäftigungsaufnahme zu beenden. Ältere haben es sehr viel schwerer, eine neue Stelle finden, wenn sie einmal arbeitslos geworden sind. Im Vergleich haben die Jüngeren zwischen 15 bis unter 30 Jahren ein höheres Zugangsrisiko in Arbeitslosigkeit, aber auch sehr viel bessere Abgangschancen aus der
Arbeitslosigkeit. Der Langzeitarbeitslosenanteil, der Anteil an den Arbeitslosen also, die länger als ein Jahr arbeitslos sind, nimmt mit dem Alter kontinuierlich zu. Drei von fünf Arbeitslosen in der Altersgruppe 50 Jahre und älter befinden sich in der Grundsicherung für Arbeitsuchende und erhalten demzufolge keine Leistungen (mehr) aus der Arbeitslosenversicherung. ${ }^{5}$ Mit der Anhebung des Rentenzugangsalters und dem Auslaufen von Alterszeit etc. wird der Anteil der Langzeitarbeitslosen in dieser Gruppe jedoch steigen.

Entgegen einer weit verbreiteten Auffassung werden der demografische Wandel und ein „Fachkräftemangel“ nicht generell zu besseren Chancen von Älteren auf dem Arbeitsmarkt und altersgerechten Arbeitsbedingungen führen. Im Zuge des demografischen Wandels nimmt zwar das Erwerbspersonenpotenzial weiter ab. Dies hat jedoch nicht zwangsläufig eine generell größere Nachfrage nach Arbeitskräften zur Folge. Erfahrungen zeigen, dass Ältere ebenso wie Langzeitarbeitslose und Menschen mit Behinderungen kaum bis gar nicht von wirtschaftlichen Aufschwüngen profitieren. Selbst für gut Qualifizierte sind die Chancen auf dem $\mathrm{Ar}$ beitsmarkt nicht durchgängig als positiv zu bewerten. Nach einem aktuellen Forschungsbericht des Instituts für Arbeitsmarkt- und Berufsforschung (IAB) hat die Mehrheit der Betriebe in absehbarer Zukunft keine offenen Fachkräftestellen zu besetzen. Wenn es überhaupt einen Fach-

1 Garloff, A./Pohl, C./Schanne, N. (2012): Demografischer Wandel der letzten 20 Jahre - Alterung der Bevölkerung hat sich kaum auf die Arbeitslosigkeit ausgewirkt: IABKurzbericht 10/2012, S. 3.

2 Statistisches Bundesamt (2009): Bevölkerung Deutschlands bis 2060, 12. koordinierte Bevölkerungsvorausberechnung, Wiesbaden, S. 19.

3 Bundesagentur für Arbeit (2012): Arbeitsmarktberichterstattung: Der Arbeitsmarkt in Deutschland, Ältere am Arbeitsmarkt, März, S. 11.

4 Vgl. BGH vom 24.04.2012 - II ZR 163/10. Zum Problem der Altersdiskriminierung vgl. auch den Beitrag von Zeibig in diesem Heft.

5 Bundesagentur für Arbeit (2012): Der Arbeits- und Ausbildungsmarkt in Deutschland - Monatsbericht, Dezember, Nürnberg, S. 88. 
kräftemangel gibt, profitiert davon nur eine gut qualifizierte Minderheit von Arbeitnehmerinnen und Arbeitnehmern in wenigen Branchen. ${ }^{6}$

\section{Förderung der Beschäftigungsfähigkeit als Zukunftsaufgabe der Arbeitsmarktpolitik}

Wenn die Arbeitsmarktintegration von Arbeitsuchenden verbessert werden soll, dann gehört es zu den Schlüsselaufgaben, der Entwertung von Qualifikationen vorzubeugen und die Beschäftigungsfähigkeit gezielt zu fördern, damit Arbeitnehmerinnen und Arbeitnehmer auch bei Verlust des Arbeitsplatzes auf dem ersten Arbeitsmarkt vermittelbar bleiben. Der Arbeitsmarkt muss durch verstärkte Bildungsanstrengungen, gezielte aktive Arbeitsmarktpolitik und den Abbau von Altersdiskriminierung für Ältere und Leistungsgewandelte weit geöffnet werden. Die bisherigen Wirkungen arbeitsmarktpolitischer Instrumente zur Förderung älterer Menschen sind dem berühmten „Tropfen auf den heißen Stein“ vergleichbar, was aber nicht gegen ihren Ansatz, sondern einzig gegen ihre Reichweite spricht.

Neben den allgemeinen Förderungsmöglichkeiten und regionalen Projekten gibt es besondere Maßnahmen zur Förderung Älterer auf dem Arbeitsmarkt. Aus der Initiative „50plus“ entstand seit 2007 die „Perspektive 50plus - Beschäftigungspakte für Ältere in den Regionen“. Hier handelt es sich um ein Programm des Bundesministeriums für Arbeit und Soziales zur Verbesserung der Beschäftigungschancen älterer Langzeitarbeitsloser. Die zweite Projektphase der „Perspektive 50+“ wurde vom Institut Arbeit und Qualifikation (IAQ) der Universität Duisburg-Essen und dem Institut für Angewandte Wirtschaftsforschung (IAW) in Tübingen evaluiert. Die Ergebnisse zeigen: Die Grundsicherungsstellen (Jobcenter) konnten im Jahr 2010 verstärkt individuell fördern, die Leistungsberechtigten nach dem Sozialgesetzbuch, Zweites Buch (SGB II) bei der Arbeitssuche begleiten, mit Coaching und Bewerbungstraining helfen - und damit deutlich bessere Eingliederungsquoten gegenüber dem Regelgeschäft erreichen.?

Über das von den Gewerkschaften angeschobene Programm „Weiterbildung Geringqualifizierter und beschäftigter Älterer in den Unternehmen“" (WeGebAU), das 2012 verstetigt wurde, ${ }^{8}$ können Arbeitnehmerinnen und Arbeitnehmer ab 45 Jahre in kleineren und mittleren Unternehmen bei ihrer beruflichen Weiterbildung durch Übernahme der Weiterbildungskosten gefördert werden. Voraussetzungen sind, dass die Weiterbildung notwendig ist, um sie bei Arbeitslosigkeit beruflich einzugliedern, eine ihnen drohende Arbeitslosigkeit abzuwenden oder weil wegen eines fehlenden Berufsabschlusses die Notwendigkeit der Weiterbildung anerkannt ist. Die Förderung ist nur möglich, wenn die Bildungsmaßnahme außerhalb des Betriebes, dem die zu Fördernden ggf. angehören, durchgeführt wird. Außerdem dürfen nur solche Kenntnisse und Fertigkeiten vermittelt werden, die über ausschließlich arbeitsplatzbezogene und kurzfristige Anpassungsqualifizierungen hinausgehen.
Der Gesetzgeber wollte damit sicherstellen, dass vorrangig im betrieblichen Interesse liegende Weiterbildungen weiterhin von den Unternehmen finanziert und Mitnahmeeffekte bei der Förderung vermieden werden. Dies steht in einem gewissen Widerspruch dazu, dass Ältere tendenziell einen hohen Praxisbezug beim Lernen und an den individuellen Lernbedürfnissen ausgerichtete Lernformen bevorzugen. Die Bildungsanbieter sind gefordert, mehr altersgerechte Lernformen anzubieten.

Programme erreichen immer nur wenige Menschen. Nachhaltig könnte hingegen eine lebenslauforientierte Arbeitsmarktpolitik wirken, die vorbeugend gegen den Verlust der Beschäftigungsfähigkeit wirkt und vor den gesundheitlichen Beeinträchtigungen durch Arbeitslosigkeit schützt. Beschäftigungsfähigkeit bedeutet mehr als die Arbeitsfähigkeit von Beschäftigten am konkreten Arbeitsplatz. Sie umfasst auch die Vermittelbarkeit auf dem allgemeinen Arbeitsmarkt. 2009 bekam die Arbeitslosenversicherung den gesetzlichen Auftrag, durch die Förderung der individuellen Beschäftigungsfähigkeit Langzeitarbeitslosigkeit vorzubeugen. ${ }^{9}$ Dieses Ziel muss auch jenseits zeitlich befristeter Programme als Handlungsauftrag begriffen und umgesetzt werden.

\section{Gute und alter(n)sgerechte Arbeit - Politik unter Zugzwang}

Die Bundesregierung hat das Thema der älter werdenden Belegschaften mit ihrer „Demografiestrategie ${ }^{\text {“10 }}$ aufgegriffen und vor allem die Unternehmen zum Handeln aufgefordert. Eigenen politischen Gestaltungswillen lässt sie jedoch vermissen. Den Unternehmen bleibt es weiterhin selbst überlassen, wie sie mit ihren Belegschaften und insbesondere älteren Beschäftigten umgehen. So finden viele Beschäftigte statt alters- und alternsgerechter Arbeitsbedingungen auch weiterhin Arbeitsverdichtung und Arbeitsdruck vor. Bei der Befragung DGB-Index Gute Arbeit 2011 sagten $63 \%$, es treffe voll und ganz bzw. eher zu, dass sie in den

6 Bechmann, S./Dahms, V./Tschersich, N./Frei, M./Leber, U./ Schwengler, B. (2012): Fachkräfte und unbesetzte Stellen in einer alternden Gesellschaft - Problemlagen und betriebliche Reaktionen: IAB-Forschungsbericht 13/2012, S. 89.

7 IAQ/IAW (2012): Evaluation der Zweiten Phase des Bundesprogramms "Perspektive 50plus" - Beschäftigungspakte für Ältere in den Regionen (2008 bis 2010), Abschlussbericht vom 30. September, S. Vff.

8 Vgl. http://www.arbeitsagentur.de/nn_508552/zentralerContent/A05-Berufl-Qualifizierung/A052-Arbeitnehmer/ Allgemein/Weiterbildung-WeGebAU.html

9 Vgl. § 1 Abs. 1 Satz 2 SGB III.

10 Vgl. Bundesregierung (2012): Jedes Alter zählt - Demografiestrategie der Bundesregierung, S. 16ff. 
letzten Jahren in der gleichen Zeit immer mehr schaffen müssten. Bei den 46- bis 55-Jährigen stimmen dieser Aussage sogar $67 \%$ zu. ${ }^{11}$ Arbeit jedoch, bei der die Beschäftigten dauerhaft unter Arbeitsverdichtung und Arbeitsdruck leiden, verbraucht die gesundheitlichen Ressourcen und ist nicht alternsgerecht. Ein von der Friedrich-Ebert-Stiftung in Auftrag gegebenes Gutachten resümiert: „Aufgrund struktureller Schwächen der Rechtsdurchsetzung mangelt es den Arbeitnehmerschutzvorschriften des Arbeitsrechts an Effektivität - und zwar häufig gerade dort, wo sie am wichtigsten wären, nämlich in Betrieben und Unternehmen, die gewerkschaftlich schwach organisiert sind und in denen es keine betriebliche Vertretung gibt. Die Beachtung von Mindeststandards für Erwerbsarbeit und Beschäftigung ist eine Frage der Menschenrechte; ihre Missachtung birgt gesellschaftliche Risiken für Demokratie und sozialen Zusammenhalt". ${ }^{\prime \prime}$

Zur Erhaltung der Beschäftigungsfähigkeit der Arbeitnehmerinnen und Arbeitnehmer sind daher konkrete und verpflichtende Regelungen notwendig. Arbeit muss so organisiert werden, dass Menschen so gesund wie möglich älter werden können. Die Rahmenbedingungen müssen durch die Politik gestaltet werden - zu moderieren reicht nicht aus.

\section{Tarifverträge und Betriebsvereinbarungen im ver.di-Organisationsbereich ${ }^{13}$}

Fällt die Politik weitestgehend als Akteur aus, gewinnen die Auswirkungen des demografischen Wandels als Gegenstand von Tarifverhandlungen und von Betriebs- und Dienstvereinbarungen zwangsläufig an Bedeutung. Die Gewerkschaften und betrieblichen Interessenvertretungen kommen mit ihren Forderungen zur Begleitung demografisch bedingter Veränderungen jedoch meist erst dann zum Zuge, wenn die Probleme sich zuspitzen und ein Handeln des Arbeitgebers erzwingen. Selbst in Branchen mit hohen physischen und psychischen Belastungen ist es für Arbeitgeber nicht selbstverständlich, sich auf einen Dialog über alters- und alternsgerechte Arbeitsgestaltung einzulassen. Dies zeigt die Weigerung des Handelsverbandes Deutschland, trotz Bildung eines Branchennetzwerkes mit ver.di im Rahmen eines vom Bundesministerium für Arbeit und Soziales geförderten
Demografie- und Tarifprojektes, ${ }^{14}$ alternsgerechte Arbeitsbedingungen tariflich zu gestalten.

Die Unternehmen haben insbesondere dann Interesse an alters- und alternsgerechten Arbeitsbedingungen, wenn sie Fachkräfte halten müssen oder sie sich aufgrund von Kündigungsschutz und Beschäftigungssicherungstarifverträgen nicht ohne Weiteres von ihren leistungsgewandelten Mitarbeiterinnen und Mitarbeitern trennen können. Altersund alternsgerechte Arbeitsbedingungen sind umgekehrt dort kaum anzutreffen, wo die Arbeitgeber aufgrund eines großen Arbeitskräfteangebots, eines hohen Restrukturierungsdrucks oder/und vergleichsweise geringer Möglichkeiten zur Beschäftigungssicherung bzw. deren Durchsetzbarkeit durch betriebliche Interessenvertretungen und Gewerkschaften geringe Anreize haben, sich um den Erhalt der Beschäftigungsfähigkeit ihrer Mitarbeiterinnen und Mitarbeiter zu bemühen. In einem deregulierten Arbeitsmarkt sind Arbeitnehmerinnen und Arbeitnehmer austauschbar und ersetzbar.

\section{Tarifvertragliche Regelungen und sozial- partnerschaftliche Vereinbarungen}

Im Dienstleistungssektor konnten erste Tarifverträge abgeschlossen werden, die speziell die Auswirkungen des demografischen Wandels betreffen. Eine umfassende Regelung enthält der „Schutz- und Generationenvertrag“ zwischen ver.di und der Deutschen Post AG vom November 2011 und mit einer Geltungsdauer bis Ende 2017. Es handelt sich dabei um ein Paket von acht Tarifverträgen und fünf weiteren Vereinbarungen für die rund 130.000 Arbeitnehmerinnen und Arbeitnehmer der Deutschen Post AG. Die Regelungen zu altersgerechtem Arbeiten umfassen ein Zeitwertkonto, Altersteilzeit und einen Demografiefonds. Ergänzt werden sie u.a. durch „AGG-konforme“ Urlaubsstaffelung nach der Betriebszugehörigkeit, die Neugestaltung des Lohnsystems und eine unbefristete Übernahmegarantie für Auszubildende nach bestandener Prüfung. Mit diesem in der Tariflandschaft einmaligen Regelungswerk haben die Tarifvertragsparteien den Auswirkungen des demografischen Wandels vor allem für die Postzustellenden Rechnung getragen, von denen $75 \%$ die Frage aus der DGB-Index-Befragung zum Erreichen
11 Ergebnisse der Repräsentativumfrage 2011 unter: http://www. dgb-index-gute-arbeit.de/jaehrliche_repraesentativerhebung/ dgb-index_gute_arbeit_2011

12 Friedrich-Ebert-Stiftung (Hrsg.) (2012): Verantwortung braucht Transparenz - Die rechtliche Verankerung unternehmerischer Pflichten zur Offenlegung von Arbeits- und Beschäftigungsbedingungen, WISO-Diskurs Mai, S. 8.

13 Tarifverträge und (Gesamt-)Betriebs- und Dienstvereinbarungen zu Lebensarbeitszeitkonten, wie sie z.B. ver.di mit dem Zentralverband der Seehafenbetriebe e.V. geschlossen hat, sowie zur Altersteilzeit bleiben hier außer Betracht.
14 Bei der Auftaktkonferenz für das Projekt „ZusammenWachsen - ArbeitGestalten" am 20. Juni 2012 in Berlin wurden Branchennetzwerke in fünf verschiedenen Dienstleistungsbranchen gegründet: Handel, Pflege, ÖPNV, Erziehungsund Sozialdienste und Straßenmeistereien, vgl. http:// www.zusammenwachsen-arbeitgestalten.de/ und http:// www.inqa.de/DE/Mitmachen-Die-Initiative/Foerderprojekte/ Projektdatenbank/zuwags-zusammenwachsenarbeitgestalten-demografie-und-tarifprojekt.html 
des Rentenalters verneinten. ${ }^{15}$ Sie stehen exemplarisch für viele Dienstleistungsberufe, in denen gesundheitliche Belastungen am Arbeitsplatz noch immer tendenziell unterschätzt werden.

Dazu gehört auch der Gesundheitsdienst. Im „Tarifvertrag zur Förderung der betrieblichen Gesundheit und der altersgerechten Arbeit - TV Gesundheitsschutz" mit der Damp Holding AG aus der HELIOS Kliniken Gruppe konnten detailliert Zusammensetzung und Aufgaben betrieblicher Gremien, die Pflicht zur jährlichen Vorlage eines Gesundheitsberichtes, Schulungen und konkrete Maßnahmen „zur Förderung und Sicherung altersgerechter Arbeit“ geregelt werden. Ein weiteres Beispiel sind der öffentliche Personennahverkehr sowie Unternehmen der Ver- und Entsorgung. ${ }^{16}$

In diesem Jahr gelang ver.di der Abschluss des „Zukunftstarifvertrages“ mit der ING-DiBa AG. Bemerkenswert ist, dass die „Erhöhung der Beschäftigungsfähigkeit“ mit interner hauptberuflicher oder nebenberuflicher Ausbildung für Arbeitnehmer ohne passende Berufsausbildung mit Lohfortzahlung und Kostenübernahme bzw. Kostenerstattung bei erfolgreichem Abschluss gefördert werden soll. Ergänzt wird dies durch Freistellungsregelungen für vom Arbeitgeber geförderte Aus- und Weiterbildungen und duale Studiengänge. Das Unternehmen sieht sich auch bei der Berufsorientierung, bei Schul- und Studierenden-Praktika und bei der Betreuung wissenschaftlicher Arbeiten in der Verantwortung. Für bürgerschaftliches Engagement ist Freistellung vorgesehen. Es gibt umfassende Regelungen zu Diversity, Gesundheitsförderung, Vereinbarkeit von Beruf und Familie, Arbeitsplatzsicherung, Altersteilzeit und zum demografischen Wandel im Allgemeinen, z.B. keine altersdiskriminierende Einstellungspraxis, Förderung von lebenslangem Lernen, alters- und hierarchieunabhängige Weiterbildung für alle und Entwicklung zukunftsorientierter Arbeitszeitmodelle unter Ausgestaltung durch Betriebsparteien.

Sind Tarifverträge nicht durchsetzbar, wird vereinzelt auf gemeinsame Erklärungen zurückgegriffen. Dies birgt zweifellos die Gefahr, das Thema demografischer Wandel ohne sichtbare Konsequenzen „abzuhaken“. So ist ver.di bereits 2006 mit einem Entwurf für einen Tarifvertrag zur Regelung gesundheitsförderlicher Arbeitsbedingungen in die Tarifrunde Banken gegangen. Erst 2010 ließen sich der Arbeitgeberverband des privaten Bankgewerbes (AGV Banken) und die Tarifgemeinschaft öffentlicher Banken auf eine „Gemeinsame Erklärung Betrieblicher Gesundheitsschutz im Bankgewerbe“ ein. Auch im öffentlichen Dienst greifen die Arbeitgeber aus Scheu vor verbindlichen Regelungen gerne auf gemeinsame Erklärungen zurück. ${ }^{17}$

\section{Regelungsgegenstände zur Gestaltung des demografischen Wandels}

(1) Altersstruktur- bzw. Demografieanalyse: Für die erfolgreiche Gestaltung des demografischen Wandels im Unternehmen bedarf es einer soliden empirischen Datenbasis, auf deren Grundlage Annahmen getroffen werden können und eine Diskussion bezüglich der richtigen Strategien geführt werden kann. Eine solche Datenbasis liefert eine Altersstrukturanalyse, auch Demografieanalyse genannt. Damit kann ein umfassendes Bild über die Zusammensetzung der Belegschaft gezeichnet werden, und zwar nicht nur entlang der Altersstruktur, sondern auch nach Merkmalen differenziert wie Geschlecht, Staatsangehörigkeit, Funktion, Qualifikation, Arbeitszeit, Betriebszugehörigkeitsdauer. Auch Erziehungs- oder Pflegeverantwortung sowie Fälle des betrieblichen Eingliederungsmanagements usw. können berücksichtigt werden. Anhand der Daten können Auffälligkeiten festgestellt, ihren Ursachen nachgegangen und Maßnahmen entwickelt werden. So können beispielweise gezielt die Anforderungen an den Erhalt der Arbeits- und Beschäftigungsfähigkeit der Belegschaft oder der Rekrutierungsbedarf für die nächsten Jahre ermittelt werden. ${ }^{18}$

Als Beispiel für die tarifliche Regelung der Altersstrukturanalyse/Demografieanalyse im Dienstleistungssektor kann der öffentliche Personennahverkehr genannt werden. So sieht der „Tarifvertrag Demografie für die Bremer Straßenbahn AG“ als Grundlage für eine „nachhaltige und vorausschauende Personalpolitik“ eine jährliche Demografieanalyse vor, die „die Klärung der personalpolitischen Ausgangssituation bei der Gesellschaft und die Ist-Analyse der Altersstruktur unter Berücksichtigung der vorhandenen Qualifikationsstruktur sowie die Belastung und Gefährdung in einzelnen Betriebsteilen“ umfasst. Durch die regelmäßige Wiederholung der Analyse ergibt sich ein Bild für bestimmte Entwicklungen im Unternehmen. Zudem verhandelt ver.di zurzeit mit der Vereinigung kommunaler Arbeitgeberverbände (VKA) über einen Tarifvertrag zum demografischen Wandel im Nahverkehr (ÖPNV). Die detaillierten Regelungen zur Demografieanalyse sind dabei im Wesentlichen unstrittig.

15 Auch 2011 wurde den Beschäftigten erneut die Frage gestellt, ob sie bei Betrachtung ihrer Arbeit und ihres Gesundheitszustandes davon ausgehen, ihre jetzige Tätigkeit bis zum Rentenalter ausüben zu können. Vor allem diejenigen zwischen Mitte 40 und Mitte 50 haben Zweifel, dass sie das Rentenalter gesund erreichen.

16 Beispiele: „Tarifvertrag Demografie für die Bremer Straßenbahn AG"; "Tarifvertrag über Arbeit und Demographie in Wasserwirtschaftsbetrieben in Nordrhein-Westfalen".

17 Als Beispiel sei die „Gemeinsame Erklärung zur demografischen Entwicklung" als "Einstieg in eine ,demografiefeste' Verwaltung zwischen ver.di und dem Land Sachsen-Anhalt genannt.

18 Zur Funktion und Durchführung der Altersstrukturanalyse s. Langhoff, Th. (2009): Den demographischen Wandel im Unternehmen erfolgreich gestalten - Eine Zwischenbilanz aus arbeitswissenschaftlicher Sicht, Dortmund, S. 53ff.; vgl. auch ver.di (Hrsg.) (2012): „Für generationsübergreifend gute Arbeit", Berlin, S. 35ff. 
(2) Übernahmeklauseln für Auszubildende: Mit der gezielten Förderung des Nachwuchses in den Unternehmen kann der demografisch bedingten „Alterung“ der Belegschaft entgegengewirkt werden. In diese Richtung wirken Übernahmeklauseln für Auszubildende. So regelt der Tarifvertrag zur Übernahme nach der Ausbildung zwischen ver.di und der IG BAU einerseits und der Arbeitgebervereinigung energiewirtschaftlicher Unternehmen andererseits in einem Zeitraum von drei Jahren die Übernahme von kalenderjährlich mindestens 200 Auszubildenden bei E.ON. Jede und jeder Auszubildende, die bzw. der die Abschlussprüfung bestanden hat, erhält einen auf mindestens ein Jahr befristeten Arbeitsvertrag.

(3) Qualifizierung: Im Laufe eines Arbeitslebens kann geringe, veraltete oder einseitige Qualifizierung zum Risikofaktor werden. Durch einen langandauernden Arbeitseinsatz in ein und derselben Tätigkeit mit geringem Anforderungswechsel und eine geringe Beteiligung an betrieblicher Weiterbildung entstehen insbesondere bei älteren Arbeitnehmerinnen und Arbeitnehmern Kompetenzlücken, die zum Veralten der beruflichen Qualifikation, Lernentwöhnung und sinkender Flexibilität führen können. ${ }^{19}$ Ältere Arbeitnehmerinnen und Arbeitnehmer nehmen neben den Geringqualifizierten erfahrungsgemäß nur unterdurchschnittlich an Weiterbildung teil. Auch insgesamt stagnierten altersspezifische Personalmaßnahmen in den vergangenen Jahren auf einem geringen $\mathrm{Ni}$ veau. Überwiegend sind diese zudem auf ein frühzeitiges Ausscheiden gerichtet. Maßnahmen, welche die spezifischen Bedürfnisse älterer Arbeitnehmerinnen und Arbeitnehmer berücksichtigen und ein gesundes Arbeiten bis zum Renteneintrittsalter unterstützen sollen, spielen nach wie vor eine geringe Rolle. Eher noch werden Ältere in bestehende Maßnahmen im Betrieb eingebunden, als dass speziell für sie Maßnahmen konzipiert werden. ${ }^{20}$

Der zwischen ver.di und dem privaten Versicherungsgewerbe geschlossene „Tarifvertrag zur Qualifizierung“ regelt die berufliche Weiterbildung im Rahmen des jeweiligen Aufgabengebietes sowie von Anpassungs- und Aufstiegsqualifizierungen. Es besteht danach ein Anspruch auf ein regelmäßiges Gespräch mit dem Arbeitgeber über den Qualifizierungsbedarf in diesem Rahmen. $\$ 5$ des Tarifvertrages für den öffentlichen Dienst (TVöD) nennt als Ziele ein hohes Qualifikationsniveau und lebenslanges Lernen, bezieht sich aber ebenso auf die jeweilige Arbeitsaufgabe. Die Förderung der Beschäftigungsfähigkeit sehen die Arbeitgeber offensichtlich nicht als ihre Aufgabe an. Die Regelung altersgerechter Lernformen ist noch tarifliches Neuland.

(4) Gesundheitsförderung: Bei Tarifverträgen zum betrieblichen Gesundheitsschutz reicht die Reglungstiefe von relativ allgemeinen Vereinbarungen (z.B. im Tarifvertrag der Evangelischen Kirche Berlin-Brandenburg-schlesische Oberlausitz - TV-EKBO), Regelungen, die arbeitsschutzrechtliche Bestimmungen vertiefen (z.B. Manteltarifvertrag für Angestellte des
Zeitschriftenverlagsgewerbes in Hamburg, Schleswig-Holstein und Mecklenburg-Vorpommern) bis zu detailliert geregelten Verfahrensfragen (z.B. Tarifvertrag zur betrieblichen Gesundheitsförderung im Sozial- und Erziehungsdienst). Der zuletzt genannte Tarifvertrag regelt u.a. den Anspruch der Beschäftigten auf die jährlich oder bei wesentlicher Änderung der Arbeitsumstände individuell durchzuführende Gefährdungsbeurteilung, mit der die mit dem konkreten Arbeitsplatz verbundenen physischen und psychischen Belastungen ermittelt werden sollen. Eine betriebliche Kommission ist zu bilden, der der Arbeitgeber die Personalplanung „hinsichtlich der insbesondere aufgrund des demografischen Wandels zu ergreifenden Maßnahmen“ vorzulegen hat.

In der öffentlichen Verwaltung finden sich Vereinbarungen unterhalb von Tarifverträgen.

\section{Betriebs- und Dienstvereinbarungen}

Auf betrieblicher Ebene unterstützen die Gewerkschaften Betriebs- und Personalräte beim Abschluss von am demografischen Wandel orientierten Vereinbarungen. Notwendige Instrumente werden an die Hand gegeben, damit maßgeschneiderte Vereinbarungen entwickelt werden können. Der erste Schritt ist immer, die betriebliche Situation zu analysieren, vorhandende Regelungen und Daten auszuwerten, Maßnahmenfelder zu identifizieren und geeignete Maßnahmen zu entwickeln. Für Konzepte im Zusammenhang mit altersund alternsgerechter Arbeit und deren Fixierung in Betriebsoder Dienstvereinbarungen gibt es weder ein Patentrezept noch eine Mustervereinbarung. Dennoch muss „das Rad nicht immer neu erfunden werden", denn es gibt mittlerweile eine größere Zahl von Vereinbarungen zu verschiedenen Themen im Kontext mit alters- und alternsgerechter Arbeit ${ }^{21}$ in den Maßnahme-Feldern Prävention, Wiedereingliederung, Arbeitsschutz/Ergonomie, Gesundheitsförderung, Qualifizierung/Personalentwicklung sowie Arbeitsorganisation/ Arbeitsgestaltung. Arbeitsorganisation und -gestaltung umfasst Änderungen in den Arbeitsabläufen, familiengerechte Arbeitsbedingungen, Wissenstransfer, Strategien gegen belastende Arbeitsbedingungen sowie alternsgerechte Berufsverläufe.

Einen ganzheitlichen Ansatz verfolgt hier die Berliner Stadtreinigung (BSR) beim Einsatz im Leistungsbereich der Straßenreinigung und Müllabfuhr. Mit Papier-

19 Vgl. Buck, H./Kistler, E./Mendius, H. G. (2002): Demografischer Wandel in der Arbeitswelt - Chancen für eine innovative Arbeitsgestaltung, Stuttgart, S. 106.

20 Vgl. Bechmann, S./Dahms, V./Tschersich, N./Frei, M./Leber, U./Schwengler, B., a.a.O. (Fußnote 6) S. $46 f$.

21 Themenbezogen enthält das Archiv der Hans-Böckler-Stiftung „Betriebliche Vereinbarungen“ Textauszüge aus betrieblichen Vereinbarungen, vgl. http://www.boeckler. de/595.htm. 
korbwerkstatt und den BSR-Scouts für außerplanmäßige Reinigungseinsätze wird ermöglicht, dass nach jahrelangem Einsatz in der Müllabfuhr von gesundheitlichen Einschränkungen betroffene Beschäftigte ihrem Leistungsvermögen entsprechend eingesetzt werden können, anstatt auf Schonarbeitsplätze abgeschoben oder aus dem Betrieb gedrängt zu werden. Zudem wurde ein Präventionsteam geschaffen, das eng mit der Fachkraft für Arbeitssicherheit und betriebsärztlichem Dienst zusammenarbeitet. Das Angebot umfasst Gesundheits- und Sozialberatung, Sport und Ernährungskurse und Konflikt- und Schuldnerberatung. Wie mittlerweile immer mehr Unternehmen, hat die BSG ein nachhaltiges Betriebliches Eingliederungsmanagement (BEM) etabliert. ${ }^{22}$

Die BSG hat mit Papierkorbwerkstatt und BSC-Scouts einen großen Schritt für altersgerechte Arbeit getan. Die Weiterentwicklung dieses Ansatzes sind alternsgerechte Berufsverläufe: Grundsätzlich wird jeder Arbeitsplatz auf seine Demografie-Festigkeit hin überprüft und nicht-demografiefeste Arbeitsplätze werden umgestaltet. Auch im Hinblick darauf, dass möglicherweise die Veränderungsmöglichkeiten für Arbeitsplätze im jeweiligen Unternehmen oder der jeweiligen Verwaltung erschöpft sind, greift eine Laufbahnplanung, die den Wechsel in eine (gleich- oder höherwertige) Tätigkeit ermöglicht. Im Dienstleistungsbereich wird hier weitgehend Neuland betreten, wobei sich insbesondere im öffentlichen Dienst erste Ansätze finden. Am Beispiel von Erzieherinnen und Erziehern würde eine alternsgerechte Laufbahnplanung bedeuten, dass Überlegungen $\mathrm{zu}$ sämtlichen Lebensphasen im Beruf von der Ausbildung über den Berufseinstieg, die Familiengründungsphase bis zum Älterwerden im Beruf angestellt werden. Durch kontinuierliche Weiterbildung werden Optionen für spätere berufliche Veränderungen offengehalten, ganz gleich, aus welchen Gründen (Aufstieg, Vereinbarkeit mit Betreuungs- oder Pflegeaufgaben, gesundheitliche Einschränkungen etc.) diese erfolgen. Umstiegs- und Aufstiegsmöglichkeiten werden eröffnet, sodass es für die Erzieherinnen auch Perspektiven außerhalb der Betreuung von Kindergruppen gibt. Diese Überlegungen sind auf andere Branchen übertragbar.

22 Das BEM ist gesetzlich vorgeschrieben (§ 84 Abs. 2 SGB IX), wird in der praktischen Umsetzung jedoch höchst unterschiedlich gehandhabt.

\section{Auf dem Weg zu alter(n)sgerechter Arbeit}

Jeder Arbeitsplatz hat seine speziellen Belastungen, denen begegnet werden muss, jede Branche ihre ganz speziellen Arbeitsbedingungen, innerhalb derer Veränderungen gestaltet werden müssen. Tarifverträge können einen verbindlichen Rahmen für Veränderungen hin zu alters- und alternsgerechter Arbeit in den verschiedenen Branchen setzen und durch betriebliche Vereinbarungen ergänzt werden. Maßnahmen zum Gesundheitsschutz bzw. zur Gestaltung alters- und alternsgerechter Arbeit sind für die Unternehmen jedoch zunächst mit Kosten verbunden, die zumeist nicht zu unmittelbaren Einsparungen an anderer Stelle führen. Die Regelung solcher Sachverhalte in Tarifverträgen ist auch längst noch nicht selbstverständlich, denn sie werden als Teil der Personalarbeit - und damit jenseits der betrieblichen Mitbestimmung - als in der alleinigen Zuständigkeit des Arbeitgebers liegend gesehen. Dies führt zu einer weit verbreiteten Verweigerungshaltung der Arbeitgeber gegenüber einem gemeinsamen Vorgehen mit den Gewerkschaften, die Anforderungen demografischer Veränderungen in der Arbeitswelt ernsthaft und verbindlich zu gestalten.

Von den bisher abgeschlossenen Tarifverträgen wird nur eine Minderheit der Arbeitnehmerinnen und Arbeitnehmern in ganz bestimmten Branchen des Dienstleistungssektors erfasst. Sie sind damit kein Ersatz für politisches Handeln zur alters- und alternsgerechten Gestaltung der Arbeitswelt. Eine Politik, die es dem einzelnen Unternehmen überlässt, wie es mit der Gesundheit und Beschäftigungsfähigkeit seiner Belegschaft umgeht, ist und bleibt verantwortungslos.

\section{AUTORIN}

EVELYN RÄDER ist Referentin für Arbeitsmarkt- und Beschäftigungspolitik sowie alters- und alternsgerechte Arbeit in der ver.di-Bundesverwaltung.

@evelyn.raeder@verdi.de 